\title{
The Prevalence of Selected Modifiable Coronary Heart Disease Risk Factors in 12-Year-Old Greek Boys and Girls
}

\section{Constantin Bouziotas, Yiannis Koutedakis, Ruth Shiner, Yiannis Pananakakis, and Vasiliki Fotopoulou}

\begin{abstract}
The prevalence of 14 selected modifiable coronary heart disease (CHD) risk factors was determined in randomly selected adolescent boys $(n=117)$ and girls $(n=93)$ from provisional Greece. Based on published criteria thresholds for CHD, $45 \%$ of boys and $50 \%$ of girls exhibited three or more risk factors with time spent on "vigorous" activities, low cardiorespiratory fitness and fatness being among the most frequent in both sexes. Stronger associations were found between cardiorespiratory fitness and time spent on "vigorous" rather than "moderate-to-vigorous" activities in both boys and girls. Regression analysis indicated that energy expenditure $(P<.01)$ in boys and energy expenditure $(P<.05)$ and energy intake $(P<.01)$ in girls could alone explain about $60 \%$ of the body-fat related findings in either group. In conclusion, broadly based primary prevention strategies - aimed at children — should concentrate on reducing the overall energy intake and increasing the time spent on "vigorous" activities if future Greek adult CHD mortality is to be reduced.
\end{abstract}

\section{Introduction}

Life expectancy of adult Greeks was until recently among the highest in the world (36). The traditional Greek diet (22) and probably an active lifestyle (35) may have accounted for this phenomenon. However, contemporary epidemiological evidence shows that mortality from CHD in Greece is beginning to increase rapidly (20). This is in line with a decrease in physical activity levels and changes in the traditional dietary habits (19) in Greek adults.

Atherosclerosis, the underlying cause of CHD, begins early in life and progresses into adulthood (33). Both unmodifiable (e.g., heredity, race, age, sex)

Constantin Bouziotas and Ruth Shiner are with the School of Health Sciences, University of Wolverhampton, WV1-1DJ, England, UK; Yiannis Koutedakis is with T.E.F.A.A., Thessaly University, Trikala, Greece; Yiannis Pananakakis is with S.E.G.A.S., Sports Medicine Center, Athens, Greece; and Vasiliki Fotopoulou is at Parmenionos 10, 60100 Katerini, Greece. 
and modifiable (e.g., physical activity, cardiovascular fitness, obesity, serum lipids, etc.) CHD risk factors are aetiologically related to atherosclerotic process and are important in their causative relationship to CHD in adult populations (5). However, modifiable CHD risk factors that increase the risk for CHD in adult populations have also been observed in paediatric populations (21) including Greek children (26) living mainly in large urban areas (2). Therefore, the aim of the present study was to characterize the risk factor profile of Greek children subjected to the beneficial effects of the traditional Mediterranean lifestyle of provisional Greece.

\section{Methods}

\section{Subjects}

The population studied consisted of 117 males (aged $12.3 \pm 0.6$ years, range 11.614.4 years) and 93 females (aged $12.3 \pm 0.6$ years, range 11.5-14.9 years). They were randomly selected from all (seven) secondary schools in Katerini, Greece (out of a total number of 384 boys and 331 girls) and reported no medical history. The Research Ethics Committee of the University of Wolverhampton approved the investigation, and permission was granted from the Greek Ministry of Education. In addition, written informed consent was obtained from the subjects and their parents after full explanation of the procedure involved. All measurements were conducted in the beginning of the school year by the same investigator, following the same order of testing procedures during the P.E. lessons. Blood tests and blood pressure recordings were conducted by a team of medical doctors at the National Hospital of Katerini.

\section{Data Collection}

Anthropometric measurements. Age (accurate to 1 month) was recorded. Weight was measured to the nearest $0.5 \mathrm{~kg}$ (Seca Beam Balance 710) with subjects being lightly dressed and barefooted and standing height to the nearest $0.5 \mathrm{~cm}$ (Seca Stadiometer 208). Percentage body fat was calculated from two skinfolds (i.e., triceps and medial calf, average of two measurements) with a Harpenden (John Bull, England) calliper using the Lohman's (25) formula.

Physical activity assessment. For the assessment of physical activity, the Aaron et al. (1) physical activity recall questionnaire and a tailor made lifestyle questionnaire were utilized. Subjects were asked to indicate all physical activities they participated in at least 10 times during the past year. For these activities, more detailed information was collected regarding the frequency and duration of participation. The lifestyle questionnaire was used to record the usual number of hours spent per day on less energy demanding activities such as sleeping, studying, TV watching, etc. The metabolic cost of each activity obtained from both questionnaires was used to calculate the time spent in daily moderate-to-vigorous $(\geq 3 \&<$ 6 METs) and vigorous ( $\geq 6$ METs) physical activity (3) as well as the total daily energy expenditure.

Cardiorespiratory fitness assessment. Cardiorespiratory fitness was assessed by using the shuttle run test (23). In brief, the subjects performed a series of runs across a 20 -m track, changing direction at the end of each run to coincide with an audio signal that gets progressively faster minute by minute. Subjects start 
running at a speed of $8.5 \mathrm{~km} / \mathrm{hr}$, and speed is increased at various stages $(0.5 \mathrm{~km} / \mathrm{hr}$ every minute). Each stage of the test is made up of several shuttle runs. The actual score of the subject is the last $1 / 2$ shuttle run fully completed before the subject drops out. Maximal oxygen uptake $\left(\dot{\mathrm{VO}}_{2} \max \right)$ in $\mathrm{ml} / \mathrm{min} / \mathrm{kg}$ was then predicted from the level at which the subject stopped exercising (12).

Smoking status assessment. Smoking status was ascertained by means of a confidential questionnaire. Subjects who smoked one or more cigarettes per week were considered as smokers.

Selected dietary intakes assessment. Information on the food intake of the subjects was collected with their parents' assistance by means of a 7-day dietary diary. All subjects were provided with a dated 7-day diary and were instructed on how to keep a record of the amount and type of food consumed on 7 consecutive days. Great care was taken to record the frequency, amount, and preparation methods of the consumed food items. An estimate of portion size for the most commonly consumed food-items was made by using sets of photographs depicting the relevant foods. Using food composition tables for Greek food (34), total energy intake and its macronutrient composition were estimated.

Serum lipids. Approximately $5 \mathrm{ml}$ of venous blood was taken from the antecubital vein under local anesthesia (xylocaine) from each subject with a vacutainer following an overnight fast. A Technicon R-XT autoanalyzer was utilized for determination of serum lipids and lipoproteins. Serum total cholesterol (TC) and TG were determined by an automated enzymatic technique (CHODPAP, Boehringer Mannheim GmbH, Germany) and CPO-PAP-method (Boehringer Mannheim GmbH, Germany), respectively. Serum HDL-C concentration was measured in the supernatant after precipitation of very-low-density and low-density lipoproteins with phosphotungstenic acid (Boehringer Mannheim Kit). Low density lipoprotein cholesterol (LDL-C) concentration was calculated according to Friedewald et al. (16) formula. External quality control took place with samples from the National System of External Evaluation of Quality of the Results in Clinical Chemistry in Athens, Greece. The coefficients of variation for TC, HDL-C, and TG were $1.9,3.8$, and $3.4 \%$, respectively.

Blood pressure recording. A mercury sphygmomanometer was used for the recording of the blood pressure after the subject had been sitting quietly for at least $5 \mathrm{~min}$. The mean of two measurements of Korotkoff phase I was recorded for systolic blood pressure. The mean of two values of Korotkoff Phase IV was recorded for diastolic blood pressure (31).

\section{Criteria Thresholds for CHD Risk}

The criteria thresholds for modifiable CHD risk factors used for the boys and girls of this study appear in Table 1 . Specific details for each of these criteria are given below.

Physical activity. For physical activity, pupils were classified as "healthy" or "at-risk" according to moderate and vigorous physical activity criteria. The moderate activity criterion classified pupils as "at risk" if they reported physical activity of moderate-to-vigorous intensity ( $\geq 3$ METs $\&<6$ METs) less than 1 hour per day. This is in line with the recommendation that all young people should participate in physical activity of at least moderate intensity for 1 hour per day (9). The vigorous activity criterion classified pupils as "at-risk" if they reported less 


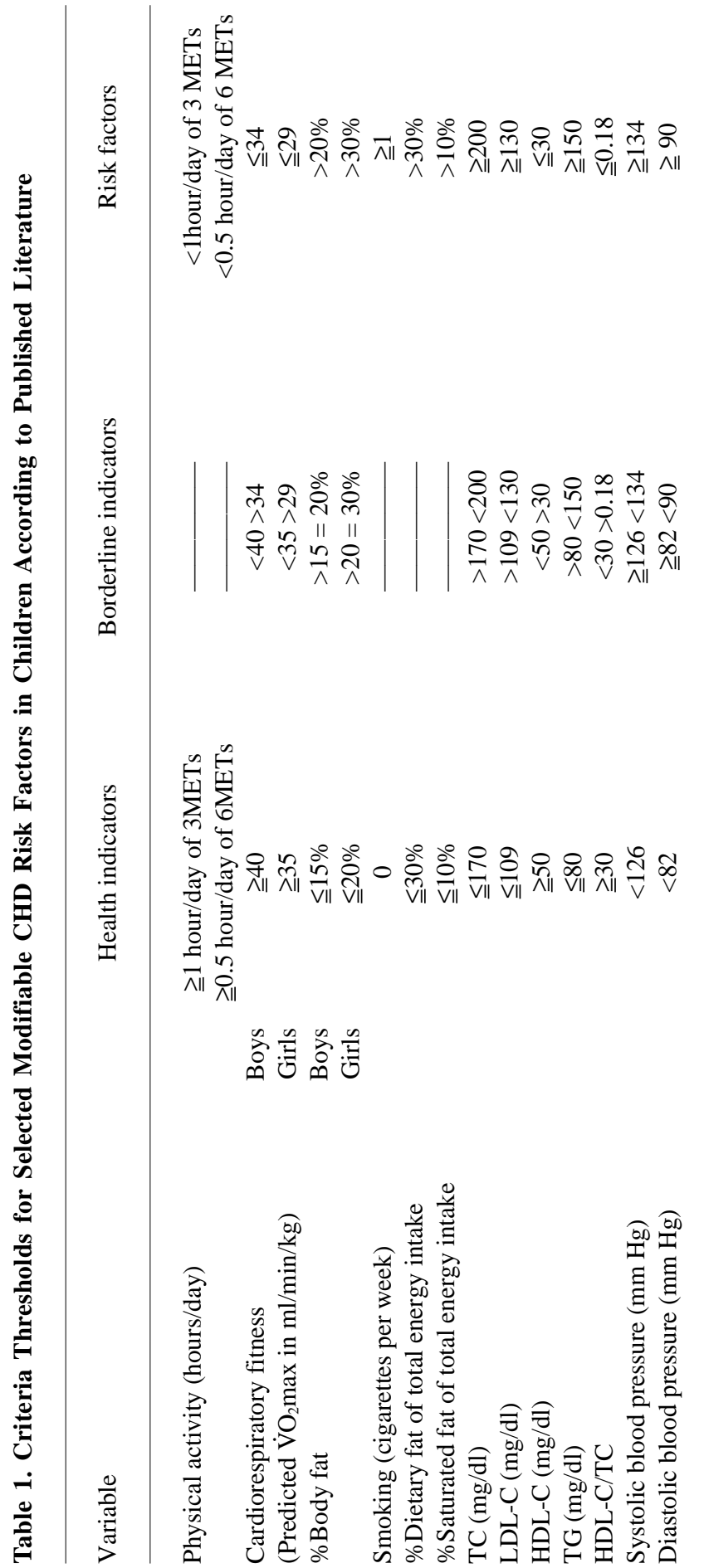


than 0.5 hour per day physical activity of vigorous intensity ( $\geq 6$ METs; 29).

Cardiorespiratory fitness. Cardiorespiratory fitness thresholds were expressed as predicted maximal oxygen uptake $\left(\mathrm{PVO}_{2} \max \right)$ in $\mathrm{ml} / \mathrm{min} / \mathrm{kg}$. On the basis of published data, all subjects were designated to one of three groups ("healthy," "borderline," or "at risk") according to criteria set by Bell et al. (7).

Body fat. All subjects were allocated into one of three groups either being "healthy," "borderline," or "at risk" according to criteria set by Bell et al. (7).

Smoking. Children who smoked at least one cigarette per week were considered to be "at-risk" as smoking even one cigarette/week is indicative of adult smoking and consequential health problems (8).

Selected dietary values. Based on published criteria for a healthy diet (28) all subjects were classified into either "healthy" or "at risk" according to their consumption of total energy intake in dietary fat and saturated fat.

Serum lipids. All subjects were allocated into one of three groups on the basis of published information $(4,7)$ : either being "healthy," "borderline," or "at risk" according to their likelihood of developing CHD.

Blood pressure. According to The Report of the Second Task Force on Blood Pressure Control in Children (31), all subjects were allocated to one of three groups either being "healthy," "borderline," or "at risk" for systolic and diastolic blood pressure, respectively.

Statistical Analyses. The SPSS statistical package version 8.0 for Windows was used for all statistical calculations. Means, standard deviations and independent $t$-tests were calculated for all parameters. Pearson correlation coefficients were calculated in order to investigate the associations between $\mathrm{PVO}_{2}$ max and time spent on "moderate-to-vigorous" and "vigorous" activities. Moreover, standard linear multiple regression analysis were also used in order to examine the relationship between energy expenditure and energy intake (independent variables) and body fat (dependent variable).

\section{Results}

The subjects' characteristics are depicted in Table 2. Indicatively, boys had better cardiorespiratory fitness $(p=0.001)$, had higher energy intake $(p=0.001)$ and energy expenditure $(p=0.001)$. However, boys were not leaner $(p \geq 0.05)$ than the girls.

Table 3 describes the population considered to be either "healthy," "borderline," or "at-risk" according to set selected modifiable criteria for CHD risk. It is worth noting that $54 \%$ and $76 \%$ of boys and girls respectively failed to exercise at intensities equivalent to or above 6 METs. Also, $54 \%$ and $52 \%$ of boys and girls respectively demonstrated low cardiorespiratory fitness with a corresponding $52 \%$ and $19 \%$ having body fat considered to be "at risk" levels.

Pearsons' correlation coefficients revealed that there are stronger associations between cardiorespiratory fitness and time spent on "vigorous" rather than "moderate-to-vigorous" activities in both boys and girls (Table 4).

Standard linear multiple regression analysis indicated that energy expenditure in boys and energy expenditure and energy intake in girls could alone explain approximately $60 \%$ of the body-fat related findings in both groups (Table 5).

Figure 1 illustrates the percentages of boys and girls with none, one, or more than one out of the 14 modifiable CHD risk factors used in this study. Only 14 boys (12\%) and 9 girls (10\%) displayed no risk factors. 


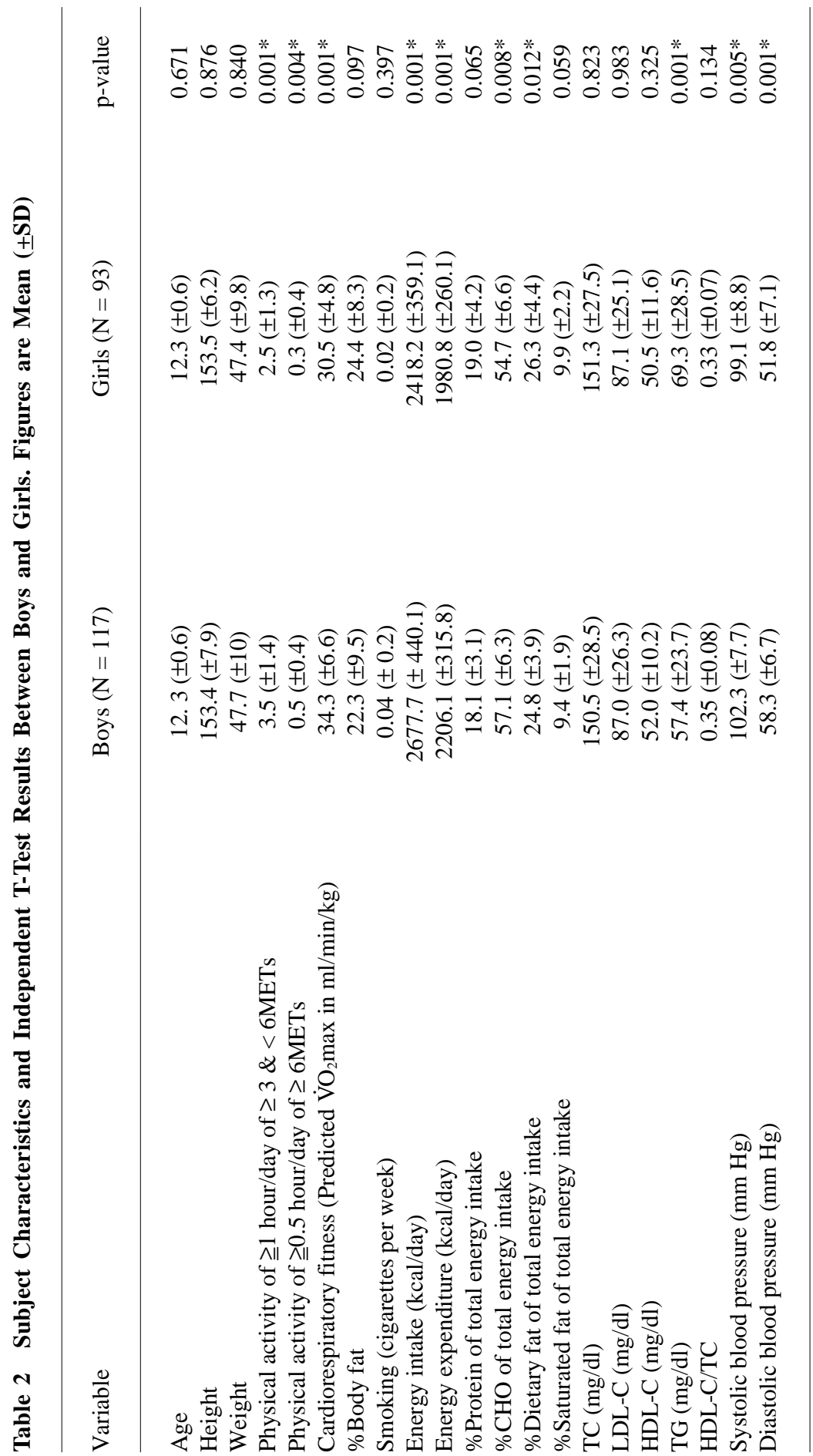




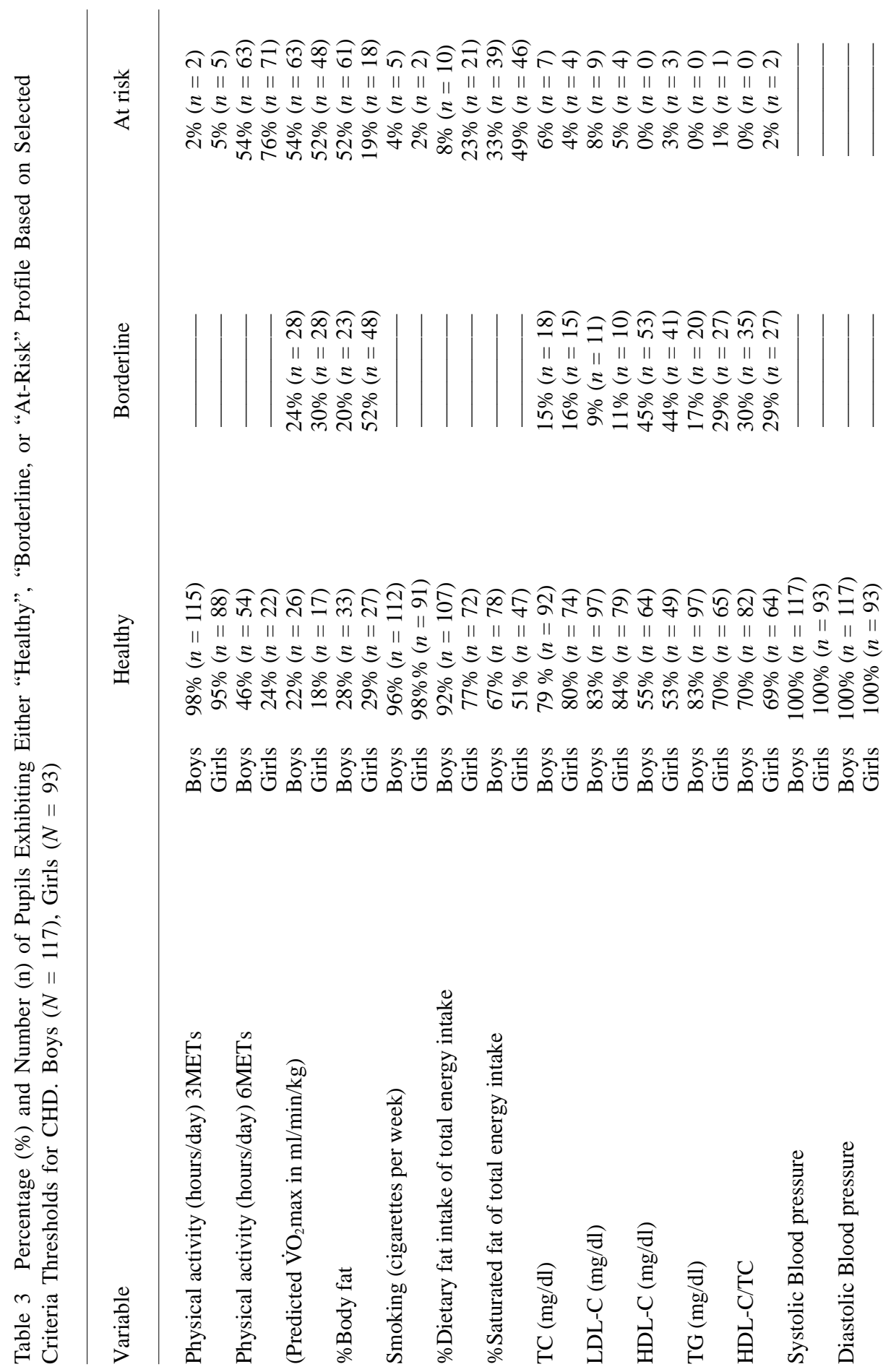


Table 4 Pearson Correlation Coefficients Between $\mathbf{P} \dot{V}_{2}$ max and Physical Activity Variables. Boys $(\mathbf{N}=117)$, Girls $(\mathbf{N}=93)$

\begin{tabular}{lcc}
\hline & \multicolumn{2}{c}{$\mathrm{PV}_{2} \max$} \\
\cline { 2 - 3 } Variables & Boys $(\mathrm{P}<0.01)$ & Girls $(\mathrm{P}<0.01)$ \\
\hline \multirow{2}{*}{ Moderate-to-vigorous physical activity } & 0.51 & 0.49 \\
Vigorous physical activity & 0.77 & 0.73 \\
\hline
\end{tabular}

Table 5 Summary of Standard Linear Regression Analysis for Variables Predicting \% Body Fat

$$
\text { Boys }(\mathrm{N}=117) \quad \text { Girls }(\mathrm{N}=93)
$$

\begin{tabular}{|c|c|c|c|c|c|c|}
\hline $\begin{array}{l}\text { Explanatory } \\
\text { variables }\end{array}$ & B & SE B & $\beta$ & B & SE B & $\beta$ \\
\hline Constant & 98.319 & 6.524 & & 76.146 & 5.006 & \\
\hline $\begin{array}{l}\text { Energy expenditure } \\
\text { (kcal/kg/day) }\end{array}$ & -1.622 & .173 & $-.764 * *$ & -.427 & .180 & $-.254^{*}$ \\
\hline \multirow{2}{*}{$\begin{array}{l}\text { Energy intake } \\
\quad(\mathrm{kcal} / \mathrm{kg} / \text { day })\end{array}$} & .003 & .137 & .002 & -.644 & .127 & $-.544 * *$ \\
\hline & \multicolumn{3}{|c|}{$\begin{array}{l}\mathrm{R}^{2}=.582, \mathrm{~F}(2,114)=79.282 \\
\mathrm{p}<.001\end{array}$} & \multicolumn{3}{|c|}{$\begin{array}{l}\mathrm{R}^{2}=.572, \mathrm{~F}(2,90)=60.230 \\
\mathrm{p}<.001\end{array}$} \\
\hline
\end{tabular}

$* \mathrm{p}<.05, * * \mathrm{p}<.01$

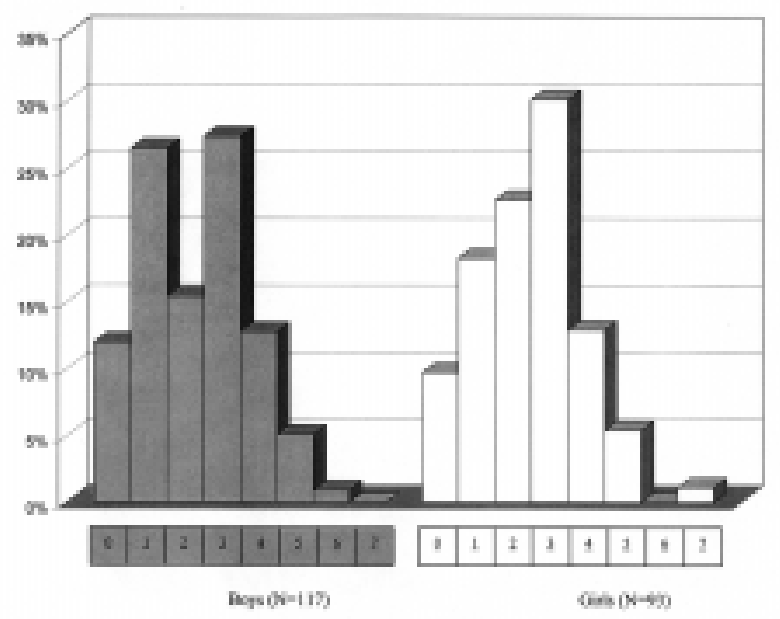

Figure 1 - Percentages of boys and girls demonstrating 0-7 out of the 14 modifiable CHD risk factors used in the present study. 


\section{Discussion}

The main findings of the present study were that only $12 \%$ of boys and $10 \%$ of girls displayed none of the commonly recognized CHD risk factors. However, more than $45 \%$ of boys and $50 \%$ of girls exhibited three or more risk factors, with lifestyle parameters such as time spent on "vigorous" (i.e., $\geq 6$ METs) activities, cardiorespiratory fitness and fatness being among the most frequent in both sexes. However, a physically active lifestyle has been found to be a crucial factor for health benefits among people of all ages (13), while attitudes toward physical activity acquired at an early age persist through adolescence into adulthood (24).

Cardiorespiratory fitness has been included among the main risk factors for CHD in children and has been linked to reduced activity levels evidenced in the Western lifestyle (10). We too found that the mean values for cardiorespiratory fitness of both boys and girls were below the health-related proposed levels for children (7). The fact that cardiorespiratory fitness levels were better served with time spent on vigorous (i.e., $\geq 6 \mathrm{METs}$ ) compared to time spent on moderate-tovigorous (i.e., $\geq 3 \&<6 \mathrm{METs}$ ) activities in our subjects further confirms the adoption of a more sedentary way of living. The need for a more active lifestyle may be explained by the fact that aerobic training effects only appear if the exercise intensities are above certain levels (6).

The present data have also shown that body fat was considered at risk levels in 52\% and $19 \%$ of our boys and girls, respectively. This finding is in line with published reports on children from other European countries (15) and from Greek children living in large urban areas $(18,26)$. Obesity is considered one of the major public health problems in industrialized countries (17). Given that mortality of obese adults, adolescents, and children is higher than that of the general population (32) and that childhood obesity predisposes for adult obesity (11), the present findings could be used to direct future public health strategies.

Regression analysis indicated that energy expenditure in boys and energy expenditure and energy intake in girls could alone explain the majority of the bodyfat related findings in both groups of the present study. These findings are in line with a recent publication suggesting that body fat accumulation in children can be prevented by regular participation in physical activities (14) and provide indirect support for the recently reported decrease in physical activity levels in Greek children (27).

The subjects of the present study reported relatively healthy dietary habits with mean fat and saturated fat intakes representing approximately $25 \%$ and $9.5 \%$ of the total daily energy intake, respectively. However, the present data contradicts the findings of Petridou et al. (30) who reported undesirable dietary patterns of children coming from urban areas of low- to middle-class Greek families. The healthy diet of the present subjects may be partly supported by their favourable serum lipid profile. Indeed, the means of all lipidemic parameters studied herein were within the health-proposed values for paediatric populations (7). These values were "healthier" than those reported by Adamopoulos et al. (2) for children living in the urban environment of the Greek capital Athens. It could be assumed, therefore, that the benefits of a more traditional Mediterranean diet might be confined to children living in provisional Greece.

In conclusion, despite a diet of relatively high quality, modifiable CHD risk factors do exist in provisional Greek children with time spent on vigorous activities, low cardiorespiratory fitness and increased fatness being among the most 
frequent. Broadly based primary prevention strategies aimed at children are essential if future Greek adult CHD mortality is to be reduced. Based on the present findings, these prevention strategies should concentrate on reducing the overall energy intake and increasing the time spent on physical activities, particularly on vigorous than on moderate-to-vigorous activities.

\section{References}

1. Aaron, D.J., A. Kriska, S.R. Dearwater, R. Anderson, T.L. Olsen, and R.R. Laporte. The epidemiology of leisure physical activity in an adolescent population. Med. Sci. Sports Exerc. 25:847-853, 1993.

2. Adamopoulos, P.N., C. Papamechael, H. Fida, N. Desses, S. Delaportas, V. Kivelos, G. Liapis, M. Iliadou-Alexandrou, and G. Kavadias. Precursors of atherosclerosis in a random sample from a Hellenic population: The Athens Study. J. Cardiovasc. Risk. 2:525-531, 1995.

3. Ainsworth, B.E., W.L. Haskell, A.S. Leon, D.R. JR. Jacobs, H.J. Montoye, J.F. Sallis, and R.S. Paffenbarger. Compendium of physical activities: classification of energy costs of human physical activity. Med. Sci. Sports Exerc. 25:71-80, 1993.

4. American Academy of Pediatrics. Statement on cholesterol. Pediatrics. 90:469-473, 1992.

5. Anding, J.D., K.S. Kubena, W.A. McIntosh, and B. O'Brien. Blood lipids, cardiovascular fitness, obesity, and blood pressure: the presence of potential coronary heart disease risk factors in adolescents. J. Am. Diet Assoc. 96:238-242, 1996.

6. Astrand, P.O., and K. Rodahl. Physiological bases of exercise. In: Textbook of work physiology. Physiological bases of exercise (3rd ed.). New York: McGraw-Hill Book Company, 1986, pp. 295-353.

7. Bell, R.D., M. Macek, J. Rutenfranz, and W.H.M. Saris. Health indicators and risk factors of cardiovascular diseases during childhood and adolescence. In: International Series on Sport Science, Vol. 17, Children and Exercise XII, J. Rutenfranz, Mocellin, and Klimt (Eds.). Champaign, IL: Human Kinetics, 1986, pp. 19-27.

8. Bewley, B. R., I. Day, and L. Ide. Smoking by children in Great Britain. A review of the literature. London: Medical Research Council and Social Science Research Council, 1972.

9. Biddle, S., J.F. Sallis, and N.A. Cavill (Eds.). Young and active? Young people and health enhancing physical activity: Evidence and implications. London: Health Education Authority, 1998.

10. Boreham, C., J. Twisk, J.M. Savage, C. Cran, and J. Strain. Physical activity, sports participation, and risk factors in adolescents. Med. Sci. Sports Exerc. 29:788-793, 1997.

11. Braddon, F.E.M., B. Rodgers, M.E.J. Wadsworth, and J.M.C. Davis. Onset of obesity in a 36 year birth cohort study. Br. Med. J. 293:299-303, 1986.

12. Brewer, J., R. Ramsbottom, and C. Williams. Multistage fitness test. A progressive shuttle-run test for the prediction of maximum oxygen uptake. Leeds, England: National Coaching Foundation, 1988.

13. Corbin, C., and R. Pangrazi. Physical activity for children. A Statement of Guidelines. Reston, VA: NASPE, 1988.

14. Dioone, I., N. Almeras, C. Bouchard, and A. Tremblay. The association between vigorous physical activities and fat deposition in male adolescents. Med. Sci. Sports Exerc. 32:392-395, 2000. 
15. Falorni, A., G. Galmacci, V. Bini, F. Papi, D. Molinari, G. De Giorgi, F. Faraoni, F. Celi, G. Di Stefano, M.G. Berioli, G. Contessa, and M.L. Bacosi. Fasting serum leptin levels in the analysis of body mass index cut-off values: are they useful for overweight screening in children and adolescents? A school population-based survey in three provinces of central Italy. Int. J. Obes. Relat. Metab. Disord. 22:1197-1208, 1998.

16. Friederwald, W.T., R.I. Levy, and D.S. Fredrickson. Estimation of the concentration of low-density lipoprotein cholesterol in plasma without the use of preparative ultracentrifuge. Clin. Chem. 18:499-502, 1972.

17. Ginsberg-Fellner, F., L.A. Jagendorf, H. Carmel, and T. Harris. Overweight and obesity in preschool children in New York City. Am. J. Clin. Nutr. 34:2236-2241, 1981.

18. Kafatos, A., G. Panagiotakopoulos, N. Bastakis, D. Trakas, M. Stoikidou, and S. Pantelakis. Cardiovascular risk factors status of Greek adolescents in Athens. Prev. Med. 10:173-186, 1981.

19. Kafatos, A., and G. Mamalakis. Policies and programmes in nutrition and physical fitness in Greece. In: World review of nutrition and dietetics: nutrition and fitness in health and disease and in growth and development, A. Simopoulos (Ed.). Karger: Basel, 1993, pp. 206-217.

20. Kalapothaki, V., A. Kalandidi, and K. Katsouyianni. The health of the Greek population (in Greek). Mater. Med. Greca. 20:91-164, 1992.

21. Katzmarzyk, P.T., R.M. Malina, and C. Bouchard. Physical activity, physical fitness, and coronary heart disease risk factors in youth: The Quebec Family Study. Prev. Med. 29:555-562, 1999.

22. Keys, A. Seven Countries: A Multivariate Analysis of Death and Coronary Heart Disease. Cambridge, MA: Harvard University Press, 1980.

23. Leger, L.A., D. Mercier, C. Gaboury, and J. Lambert. The multistage 20-m shuttle run test for aerobic fitness. J. Sports Sci. 6:93-101, 1988.

24. Livingstone, M.B.E. Energy expenditure and physical activity in relation to fitness in children. Proceedings of the Nutrition Society. 53:207-221, 1994.

25. Lohman, T.G. Advances in body composition assessment: Current issues in exercise science. Monograph Number 3. Champaign, IL: Human Kinetics Publishers, 1992.

26. Mamalakis, G., and A. Kafatos. Prevalence of obesity in Greece. Intern. J. of Obesity. 20:488-492, 1996.

27. Manios, Y., A. Kafatos, and C. Codrington. Gender differences in physical activity and physical fitness in young children in Crete. J. Sports Med. Phys. Fitness. 39:24-30, 1999.

28. NCEP. Report of the Expert Panel on Blood Cholesterol Levels in Children and Adolescents. Rockville, MD: U.S. Department of Health, Education, and Welfare; National Institutes of Health publication N4LB1, 1991.

29. Pate, R.R., S.G. Trost, M. Dowda, A.E. Ott, D.S. Ward, R. Saunders, and G. Felton. Tracking of physical activity, physical inactivity, and health-related physical fitness in rural youth. Ped. Exer. Sci. 11:364-376, 1999.

30. Petridou, E., E. Malamou, S. Doxiadis, S. Pantelakis, G. Kanellopoulou, N. Toupadaki, A. Trichopoulou, V. Flytzani, and D. Trichopoulos. Blood lipids in Greek adolescents and their relation to diet, obesity, and socio-economic factors. Ann. Epidemiol. 5:286291, 1995.

31. Report of the Second Task Force on Blood Pressure Control in Children. Task Force on Blood Pressure Control in Children. Pediatrics. 79:1-25, 1987.

32. Saw, S.M, and U. Rajan. The epidemiology of obesity: a review. Ann. Acad. Med. Singapore. 26:489-493, 1997. 
33. Strong, J.P., G.T. Malcom, C.A. McMahan, R.E. Tracy, W.P. 3rd Newman, E.E. Hederick, and J.F. Cornhill. Prevalence and extend of atherosclerosis in adolescents and young adults: implications for prevention from the Pathobiological Determinants of Atherosclerosis in Youth Study. JAMA. 24:727-735, 1999.

34. Trichopoulou, A. Food composition tables of Greek foods and dishes. Athens, Greece: Passianos, 1992.

35. Trichopoulou, A., K. Katsouyianni, and C. Gnardelis. The traditional Greek diet. Eur. J. Clin. Nutr. 47(Suppl. 1):S76-S81, 1993.

36. WHO. Annual epidemiological and vital statistics for 1960. Geneva: WHO, 1963. 